\title{
Field Performance of Winegrape Rootstocks and Fumigation during Establishment of a Chardonnay Vineyard in Washington
}

\author{
Katherine E. East,${ }^{1 *}$ Inga A. Zasada, ${ }^{2}$ Julie Tarara,${ }^{3}$ and Michelle M. Moyer ${ }^{4 *}$
}

\begin{abstract}
In Washington, most winegrapes are own-rooted Vitis vinifera, which is susceptible to the plant-parasitic nematodes Meloidogyne hapla and Xiphinema americanum. Using resistant rootstocks to manage nematodes has not been evaluated in Washington vineyards. A long-term vineyard trial was established to evaluate the effects of soil fumigation and rootstock genotype on $M$. hapla and X. americanum population dynamics and vine growth during vineyard establishment (first three years) in a replant scenario. Vines in an existing $V$. vinifera Chardonnay vineyard were first treated with foliar glyphosate in fall 2014. Randomized areas within the vineyard were then either fumigated or not with drip-applied metam sodium. Following fumigation, vines were removed. In spring 2015, the vineyard was replanted to Chardonnay on the following rootstocks: 1103 Paulsen, 101-14 Millardet et de Grasset, Teleki 5C, and Harmony. Self-grafted and own-rooted Chardonnay were included. Fumigation reduced M. hapla soil second-stage juvenile (J2) population densities on own-rooted and self-grafted vines for only the first year postfumigation. One year postfumigation, the self-grafted and own-rooted vines had higher population densities of $M$. hapla J2 than rootstocks. All rootstocks supported measurable densities of $M$. hapla $\mathrm{J} 2$ but were poor hosts relative to $V$. vinifera. Fumigation effectively reduced population densities of $X$. americanum for up to 3.5 years. Fumigation also reduced early establishment pruning weights. Vines grown in fumigated areas had lower pruning weights through year 2, but rootstock was the bigger influence on pruning weights by year 3. This trial demonstrates that rootstocks have a more sustained impact on nematode reestablishment and subsequent vine health in a vineyard replant scenario than that of preplant fumigation.
\end{abstract}

Key words: fumigation, Meloidogyne hapla, nematode dosage, rootstock, Vitis vinifera, Xiphinema

Washington grapegrowers lack basic information regarding plant-parasitic nematodes upon which to make informed pre- and postplant management decisions. As vineyards have aged and the maturing Washington winegrape industry begins a period of vineyard replant, plant-parasitic nematodes have become a greater concern for the winegrape industry. In Washington State vineyards, the nematodes of greatest im-

\begin{abstract}
${ }^{1}$ Former address: Michigan State University - Southwest Michigan Research and Extension Center, Benton Harbor, MI 49022; ${ }^{2}$ USDA-ARS-Horticultural Crops Research Laboratory, Corvallis, OR 97330; ${ }^{3}$ Ste. Michelle Wine Estates, Prosser, WA 99350; and ${ }^{4}$ Washington State University - Irrigated Agriculture Research and Extension Center, Prosser, WA 99350.

*Corresponding authors (michelle.moyer@wsu.edu; tel: 509-786-9234; fax 509-786-9370; katherine.e.east@gmail.com)

Acknowledgments: Funding for this project was provided through the Washington State Grape and Wine Research Program, including Washington State Wine Commission, Auction of Washington Wines, State Liter tax, and/or WSU Agriculture Research Center. This research was also partially funded by USDAARS Current Research Information System 2072-22000-043-00D and USDA National Institute of Food and Agriculture Hatch project 1016563. The authors thank Mimi Nye, Pedro Flores, Kari Smasne, and Melinda Garza of Ste. Michelle Wine Estates for their expertise and vineyard support. The authors also thank Ashley Boren, Catie Wram, Amy Peetz, Eric Gale, Margaret McCoy, Jensena Newhouse, Maria Mireles, and Jack Pinkerton for their technical assistance.

Supplemental data is freely available with the online version of this article at www.ajevonline.org.
\end{abstract}

Manuscript submitted April 2020, revised Sept 2020, accepted Oct 2020

This is an open access article distributed under the CC BY license (https:// creativecommons.org/licenses/by/4.0/).

By downloading and/or receiving this article, you agree to the Disclaimer of Warranties and Liability. The full statement of the Disclaimers is available at http://www.ajevonline.org/content/proprietary-rights-notice-ajev-online. If you do not agree to the Disclaimers, do not download and/or accept this article. doi: 10.5344/ajev.2020.20023 portance are the northern root-knot nematode (Meloidogyne hapla) and dagger nematode (Xiphinema americanum in the broad sense). M. hapla and Xiphinema spp. were detected in $60 \%$ of surveyed vineyards, with $20 \%$ of these over the suggested thresholds of either $100 \mathrm{M}$. hapla/250 g soil or $25 \mathrm{Xi}$ phinema spp./250 g soil (Zasada et al. 2012). If young vines are planted into infested soils, the vineyard is immediately at an establishment disadvantage; this could manifest as either young vine death or the failure to meet production goals for the duration of the vineyard's lifespan. This has been reported in other grapegrowing regions, including California and Australia (Nicol et al. 1999, Westphal et al. 2002).

In perennial crop production, the most important window for nematode control is prior to establishment, and this is often achieved with preplant soil fumigation (Zasada et al. 2010), which immediately kills target nematodes with little residual effect. Efficacy of fumigation varies depending on soil moisture, soil type, amount of nematode-infected root material left in the ground, and whether vineyard infrastructure was left in place (Lembright 1990). The long-term effect of fumigation on plant-parasitic nematode species such as M. hapla and Xiphinema spp. has not been established in vineyards. Due to changes in regulations regarding soil fumigation (https:// www.epa.gov/soil-fumigants/regulatory-status-fumigants), this practice will become more cumbersome. In addition, many growers are interested in participating in sustainable programs (e.g., Low Input Viticulture and Enology; LIVE), which do not allow soil fumigation. If nematodes are not controlled prior to planting, there are few effective postplant management options available for vineyards, including no proven effective registered postplant nematicides at the time of this publication. 
Another option available to growers for plant-parasitic nematode management is the use of resistant or tolerant grape rootstocks (Sauer 1967, Harris 1983, McKenry et al. 2001, Ferris et al. 2012, Zasada et al. 2019). Washington State winegrape growers generally grow Vitis vinifera on their own roots, due to a historical lack of established phylloxera (Daktulosphaira vitifoliae) and the threat of aboveground cold damage (Moyer et al. 2011). Because of this, there is a lack of enthusiasm for the adoption of rootstocks to manage soil-borne pests or soil-related problems, which has the compounding effect of lack of interest in evaluation of rootstocks under Washington's climate (Keller et al. 2012). However, this lack of enthusiasm has very recently changed due to vineyard survey results that indicate a more established presence of phylloxera than originally thought (Prengaman 2019). A third complication to making informed decisions on the selection of a rootstock for M. hapla management in Washington is the fact that most Meloidogyne species-resistant rootstocks have not been tested against $M$. hapla, but rather, other Meloidogyne species that are problematic in other grapegrowing regions (McKenry et al. 2001, McKenry and Anwar 2006, Ferris et al. 2012). Whether these rootstocks are also resistant to $M$. hapla in a vineyard setting remains to be determined. Although we have evaluated the host status of rootstocks for M. hapla in greenhouse experiments (Zasada et al. 2019), the applicability of shortterm rootstock evaluation trials (greenhouses, pot studies) to the reality of long-term vineyard establishment and production can be problematic (McKenry et al. 2001, McKenry and Anwar 2006, Ferris et al. 2012). Rootstocks that may appear resistant or tolerant to a nematode infestation in the short term may not be in the long term, and this might be exacerbated under field conditions.

The objectives of this research were designed to address several of the concerns related to 1 ) how preplant fumigation affects $M$. hapla and Xiphinema spp. population densities both immediately and for several years postfumigation; 2) how the chosen rootstocks affect vine growth under Washington State's climate; and 3) how effective these rootstocks are at limiting the population growth of $M$. hapla and Xiphinema spp. or tolerating nematode feeding over time. The commercial vineyard established as a part of this experiment is part of a long-term (over 10 years) trial designed to measure the duration of efficacy for preplant fumigation, as well as long-term impacts of plant-parasitic nematode infestation on vineyard health and productivity. This paper examines the establishment phase of this vineyard, defined here as the first three growing seasons, from planting in 2015 to the first partial-cropping year in 2017.

\section{Materials and Methods}

An existing $V$. vinifera Chardonnay (clone unknown) vineyard planted in 1992 in Paterson, WA $\left(45^{\circ} 87^{\prime} \mathrm{N} ;-119^{\circ} 76^{\prime} \mathrm{W}\right)$, was used for this experiment. It had established populations of M. hapla (northern root-knot nematode) and Xiphinema sp. (dagger nematode). The Xiphinema sp. found at this site belongs to the taxonomically confusing $X$. americanum species complex (Robbins 1993, Zasada et al. 2012) and has not been assigned a specific designation within this group. For the purpose of this paper, this population will be referred to as $X$. americanum. The grower had slated this site for replanting, with the intent of maintaining the existing vineyard infrastructure (e.g., retention of vineyard posts and irrigation lines). The soil at the site is a Quincy loamy sand, with a 0 to 30\% slope (Web Soil Survey; https://websoilsurvey.sc.egov. usda.gov/App/WebSoilSurvey.aspx). On 5 Sept 2014, the existing vines were treated with a foliar glyphosate $(2 \%$ solution in $702 \mathrm{~L} / \mathrm{ha}$ ) application as described by Moyer et al. (2017) (Figure 1B). Approximately two weeks after the foliar glyphosate application, the vineyard block was divided into four replicate plots of six rows each (total of 24 rows, Figure 1A). Each of the four plots was randomly assigned a fumigation treatment to one-half of the plot, where one-half of each row was fumigated and the other half was left nonfumigated. Soil fumigation consisted of metam sodium CLR $42 \%$ (sodium methyldithiocarbamate; metam sodium; Vapam HL) applied through the existing irrigation drip tubing with $1.9 \mathrm{~L}$ ( 0.5 gallon) emitters on 18 Sept 2014 at a rate of $215 \mathrm{~L} /$ ha delivered in $702 \mathrm{~L} / \mathrm{ha}$ for $8 \mathrm{hrs}$ and then flushed for $2 \mathrm{hrs}$ as described by Moyer et al. (2017). Postfumigation, the existing vines were removed (between 28 Oct and 18 Dec 2014), but the trellising infrastructure was retained; this allowed for the evaluation of the impact of fumigation in a replant scenario where the existing trellis system is retained. Retention of existing vineyard infrastructure in Washington State vineyard replant scenarios is common practice.

On 12 May 2015, the site was replanted in a randomized split-block design consisting of six main plot vine rootstock treatments, with fumigation (fumigated $=\mathrm{F}$; nonfumigated = NF) as the split-block treatments (Figure 1A). The treatments were replicated four times. Vine treatments consisted of entire rows ( 106 vines/row) planted on one of four rootstocks or nongrafted or self-grafted $V$. vinifera. The entire experimental area was $\sim 2.4$ ha. Rootstocks were chosen for their resistance to Meloidogyne species (Sauer 1967, Chitambar and Raski 1984, Nicol et al. 1999, McKenry et al. 2001, 2006, Ferris et al. 2012), as well as availability of certified stock material. All planted materials, both rootstock and scion, were certified either through the Washington State Department of Agriculture or the California Department of Food and Agriculture. The rootstocks evaluated were Millardet et de Grasset 101-14 (101-14 Mgt), Harmony, Paulsen 1103 (1103 P), Teleki 5C (5C), and a self-grafted (Chardonnay grafted to Chardonnay) control. Harmony was selected over the similar rootstock Freedom due to availability from the supplier. Chardonnay Foundation Plant Services selection 15 was the scion. A second control, nongrafted, own-rooted Chardonnay, was included in the experimental design because this is the most common form of planting material in Washington State. All grafted rootstocks and self-grafted vines were bench-grafted using an omega graft. All rootstocks, aside from Harmony, were grafted in spring of 2014, greenhouse-healed in summer and fall 2014, and placed into dormancy for the 2014 to 2015 winter. Harmony was grafted in fall 2014, and greenhouse-healed for the 2014 to 2015 winter, then shipped for planting without a dormancy period. All vines were planted the week of 11 May 2015. 
Within the NF plots, a continuous 10-vine section was inoculated with $\sim 20,000$ supplemental $M$. hapla eggs applied to the roots of vines in $10 \mathrm{~mL}$ water at planting $(\mathrm{NF}+$ ) (Figure 1D). This inoculated 10 -vine section was designed to mimic an extreme level of nematode pressure in a replant situation. M. hapla egg inoculum was produced by collecting soil from the vineyard the year prior to establishment and planting with tomato. Eggs produced on tomato roots were extracted using bleach, concentrated in water, and adjusted to the desired density. Since the NF+ treatment was limited to 10 -vine sections within each rootstock replicate row, two other continuous 10 vine sections were marked off for data collection in each row as well, one in the F block and one in the uninoculated parts of the NF block (Figure 1C). All data were collected from these 10-vine sections.

Vines were trained toward the cordon wire (1.06 $\mathrm{m}$ high) in 2015. In 2016, vines were trained to a single trunk with bi- lateral cordons. In 2017, the canopy was trained to a modified vertical shoot positioning with catch wires at $28 \mathrm{~cm}$ above the cordon wire. The vines were spur pruned in the 2017 to 2018 winter. This vineyard block is part of a commercial operation, and the grower-cooperator managed all irrigation, fertilization, and pest management practices.

Nematode sampling and processing. Soil was sampled for $M$. hapla second-stage juveniles (J2) and $X$. americanum mixed stages starting prefumigation in fall 2014 and continuing in spring and fall from spring 2015 to spring 2018 (total of eight sampling dates). At each sampling date, 10 soil cores, $2.5 \mathrm{~cm}$ diameter by $45 \mathrm{~cm}$ deep, were collected under drip emitters in each 10-vine treatment section (Figure 1) and combined into one composite sample. Previous work has shown that M. hapla in Washington drip-irrigated vineyards are concentrated under drip emitters (Howland et al. 2014) and that $X$. americanum are evenly distributed throughout a vineyard

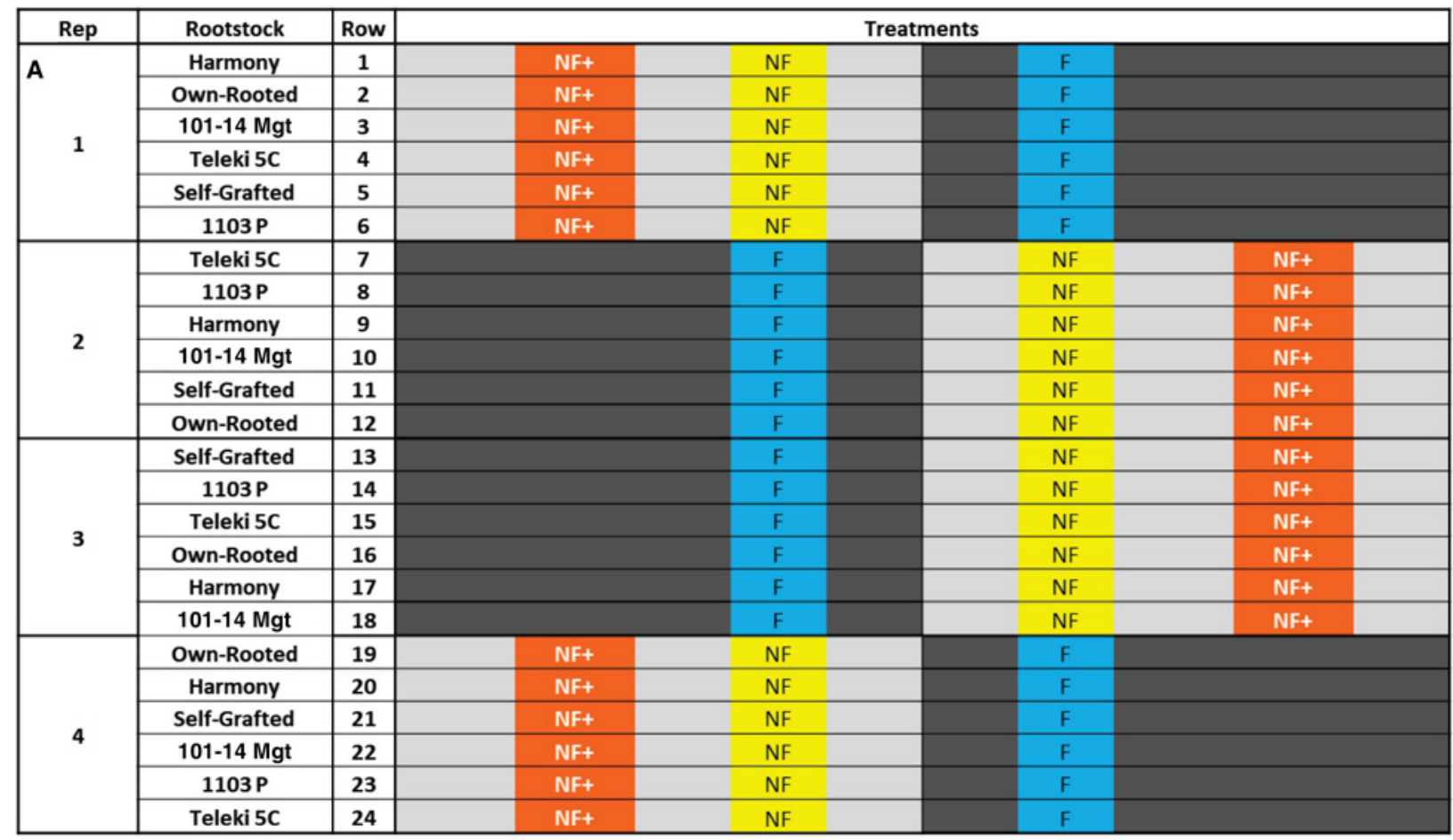
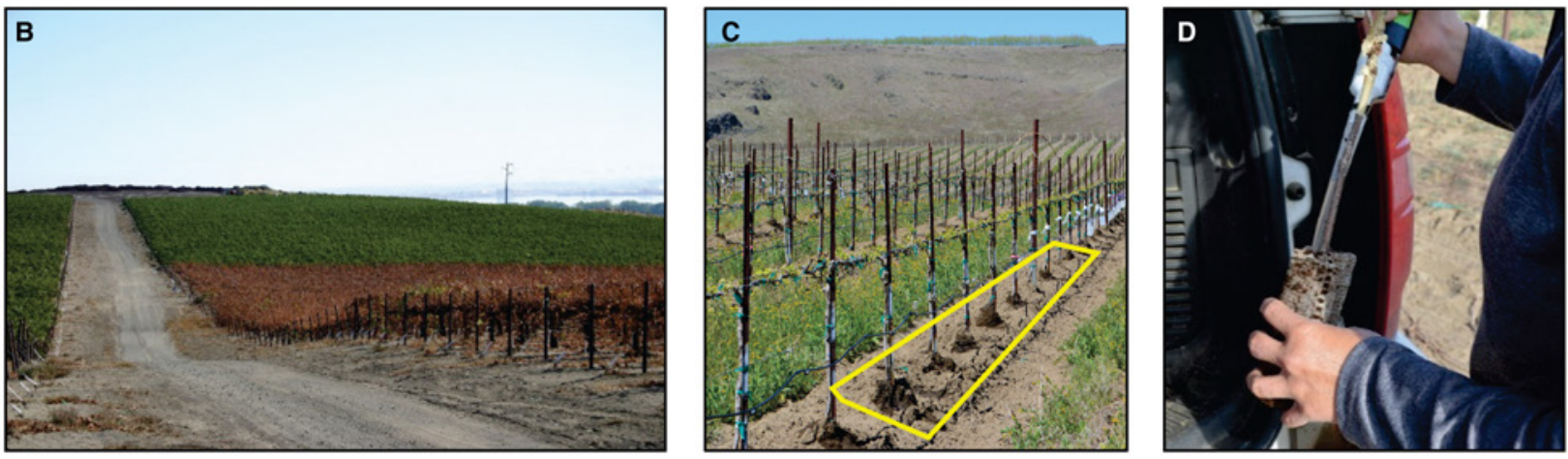

Figure 1 (A) Vineyard experimental diagram, where a rootstock consists of an entire vineyard row and where half of that row was either fumigated (dark gray) or not fumigated (light gray). Within each vineyard row, three, 10-vine sections were designated for data collection: $F$ (blue) in the fumigated half; NF (yellow), in the nonfumigated half of the row; and NF+ (orange), where $\sim 20,000$ additional Meloidogyne hapla eggs were added to vine roots at planting in the NF half of the row. Fumigation occurred on 18 Sept 2014, and planting and additional nematode inoculation occurred on 12 May 2015. (B) Foliar glyphosate was applied to kill the existing vines in fall 2014 prior to being removed and replanting. (C) Vineyard rows with NF (yellow) 10-vine section highlighted as an example. (D) In the NF+ plots, vines were inoculated with $M$. hapla eggs prior to planting. 
soil (East et al. 2019b). Soil core samples were processed using a semiautomatic elutriator (Seinhorst 1962) and further processed using a sugar centrifugation technique (Jenkins 1964) to collect $M$. hapla $\mathrm{J} 2$ and $X$. americanum. Nematodes were enumerated using a Leica DM IL inverted microscope (Leica Microsystems). Root samples were collected each fall, from 2015 to 2017 , by removing a $1000 \mathrm{~cm}^{3}$ section of soil from under three drip emitters in each treatment plot; roots were gently shaken out of soil manually and collected. Eggs were collected from root samples using a bleach method in which roots were shaken in a $10 \%$ sodium hypochlorite solution for 3 min and then poured over stacked 88 - and $25-\mu \mathrm{m}$ sieves to separate roots and eggs (Hussey and Barker 1973). Collected eggs were counted using an inverted microscope.

Vine parameters. Dormant pruning weights were used as an assessment of vine vigor. Each vine per 10-vine data plot was pruned, and pruning debris was collected and weighed on a per-vine basis. In 2015, the year of planting, vines were segregated into two categories and pruned accordingly on 17 Dec. These categories and their treatments were:

- "2-Bud": Prune the trunk back to two buds only to encourage root development and stronger vine growth for the following season;

- "Training": On vines that had established well, prune the trunk back to $10 \mathrm{~cm}$ below the fruiting wire.

Vine vigor in this year was assessed as the percentage of vines assigned to the "Training" category, rather than by pruning weight. This approach was done to account for the degree of pruning. Actual pruning weights recorded would have been the inverse of actual vine vigor; in other words, when pruning back to two buds, heavier pruning weights were often recorded than from vines that were pruned back to just below the fruiting wire. If evaluated based on weight only, those vines that were pruned back to two buds would have appeared to have better growth (high pruning weights) when in fact they did not. During the 2016 and 2017 growing seasons, all vines were trained up to and on the wire, dormant vines were spur pruned to three-bud spurs, and pruning weight per vine was recorded (vines were pruned on 14 Dec 2016 and 14 Nov 2017). In 2017, the first partial-crop was harvested on 29 and 30 Aug from the 10 -vine subplots, and individual vine yield was recorded.

Data analysis. Nematode densities of M. hapla and $X$. americanum were analyzed using the standard least squares model platform in JMP (version 14.0.0, SAS Institute, Inc.) as a split-plot with fumigation, rootstock, fumigation $\times$ rootstock, and block $\times$ rootstock, with fumigation and rootstock as fixed variables and block as a random variable. Egg data were log $(\mathrm{x}+1)$ transformed to meet assumptions of normality, and this output was analyzed as above. Where significant differences were found, mean separation was performed using Tukey's honest significant difference (HSD) test. Table outputs are included as supplemental data (Supplemental Tables 1 to 3).

In addition, M. hapla $\mathrm{J} 2$ data from fall of 2015, 2016, and 2017 were categorized within fumigation treatments and rootstock treatments to one of three categories. These categories captured densities relative to the proposed threshold of 100 M. hapla $\mathrm{J} 2$ for management (Santo, unpublished). The cat- egories were defined as Category 1: fewer than $50 \mathrm{M}$. hapla $\mathrm{J} 2 / 250 \mathrm{~g}$ soil, where a management response would not likely occur; Category 2: 50 to $150 \mathrm{M}$. hapla J2/250 g soil, where a management response is recommended but the degree of response may be tempered by other site factors; and Category 3: greater than $150 \mathrm{M}$. hapla $\mathrm{J} 2 / 250 \mathrm{~g}$ soil, where a management response would be highly recommended. At each time point, in each fumigation $\times$ rootstock treatment combination, the replicate average was used for category placement in fall 2015, 2016, and 2017.

To evaluate the impact of $M$. hapla population densities on own-rooted vine growth, the typical situation in Washington State vineyards, we analyzed just the self-grafted and ownrooted vines. $M$. hapla $\mathrm{J} 2$ enumerated from soil in fall most likely invade and impact vines in the following year (East et al. 2019a). Using the pruning weights from the following year as a measure of vegetative growth may be a better indicator of the impact of $M$. hapla $\mathrm{J} 2$ population densities on vine vigor than pruning weights of the current year, given that time needed for the impact of nematode feeding to be realized. Meloidogyne spp. density has been correlated with vine vigor (Ferris and McKenry 1975). M. hapla J2 population densities were ranked within time points, as was pruning weight. Ranked $M$. hapla J2 population densities were compared with ranked pruning weights in self-grafted and own-rooted vines at the end of the following year within each replicate (e.g., fall 2015 M. hapla J2 and fall 2016 pruning weights). Because the data were nonnormally distributed and comparisons across years with very different densities of $M$. hapla J2 and pruning weights were desired, ranking allowed for comparisons across years.

To measure the cumulative nematode pressure experienced by grapevines over multiple growing seasons, nematode dosage was calculated (Noling and Ferris 1987). Cumulative nematode dosage is calculated similarly to the area under the disease progress curve (AUDPC) and is expressed in nematode degree days (the nematode density measured over multiple sampling dates multiplied by the degree days accumulated between sampling dates). M. hapla $\mathrm{J} 2$ population densities were $\log (\mathrm{x}+1)$ transformed to fit assumptions of normality at each sampling point, spring and fall, from spring 2015 (at planting) to spring 2018 (three years postplanting). At these time points, growing degree days were calculated using base $10^{\circ} \mathrm{C}$ from soil temperatures recorded at $20 \mathrm{~cm}$ under the soil surface at a nearby weather station maintained by Washington State University (AgWeatherNet). The total area under the curve formed by $M$. hapla $\mathrm{J} 2$ population densities at each degree-day time point was the nematode dosage over that time period. Mean separation of nematode dosage was done using JMP 14.0.0 in the standard least squares platform, with block as a random effect and fumigation, rootstock, and fumigation $\times$ rootstock as fixed effects. Means were separated using the Tukey HSD test at the $0.05 \alpha$ level.

\section{Results}

Effect of fumigation on vine vigor. There was no interaction between rootstock and fumigation in any year on pruning 
weights or yield; therefore, they are presented separately in Figure 2. In 2015, after the first year of growth, direct pruning weights were not a reliable measure of vigor, as vines were pruned differently depending on how close they had grown to the fruiting wire (different pruning strategies based on vine development is a common practice in vineyard establishment).
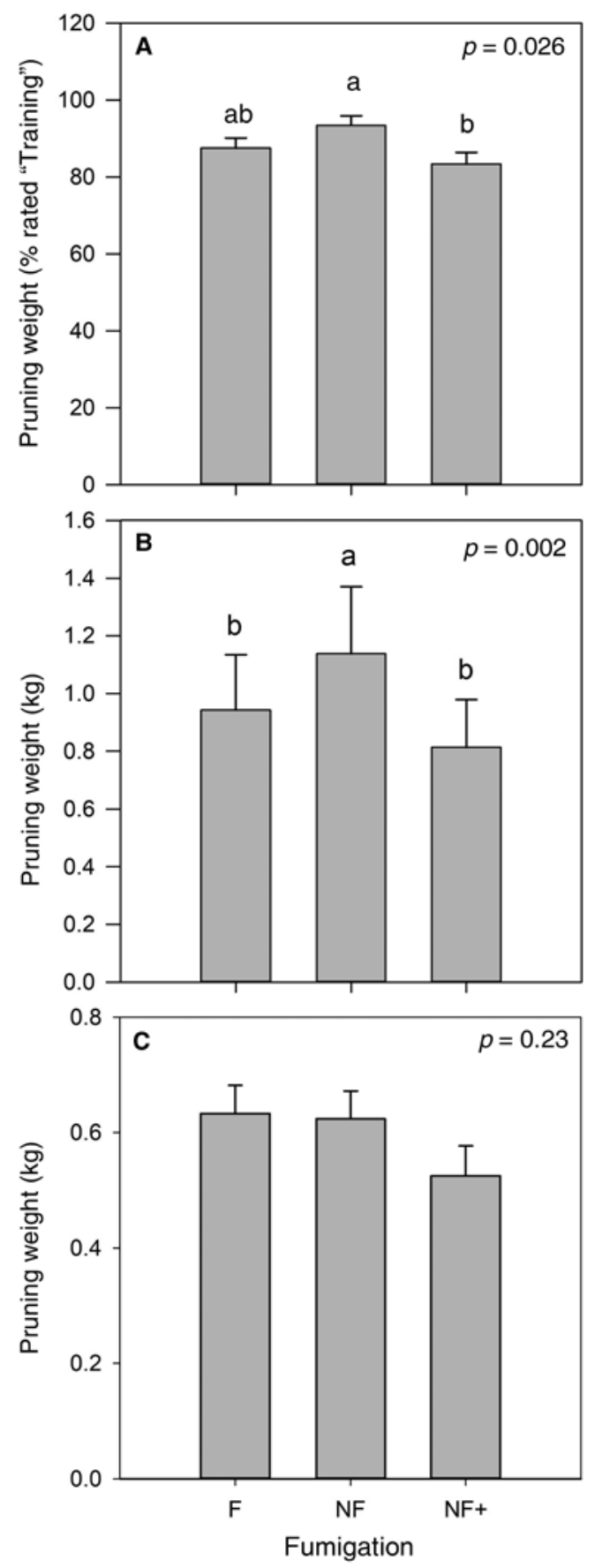

Figure 2 Dormant vine pruning weights by fumigation (soil) treatment in fall of (A) 2015, (B) 2016, and (C) 2017. Fumigation treatments were fumigated (F), nonfumigated (NF), and nonfumigated inoculated (NF+), where 20,000 additional Meloidogyne hapla eggs were added to the vines at planting. Rootstock treatments were combined within fumigation treatments because there was no rootstock $\times$ fumigation interaction. Fumigation occurred on 18 Sept 2014. Planting occurred on 12 May 2015. Error bars are standard error $(n=4)$. Different letters denote significant differences among treatment means at $\alpha=0.05$ using Tukey's honest significant difference.
Instead, they were categorized as "2-Bud" lower vigor vines, which were cut back to two buds, and "Training," higher vigor vines that had reached the fruiting wire, and so were pruned just below the fruiting wire. More vines in the NF plots were rated "Training" (higher vigor) than in the NF+ plots, and $\mathrm{F}$ plots fell in between with intermediate vigor $(p=0.026)$ (Figure 2A). Starting in 2016, pruning weights could be reliably used directly as a measure of vine vegetative vigor. In 2016 , vines in the NF plots had higher pruning weights than in both the $\mathrm{F}$ and NF+ plots $(p=0.002)$ (Figure 2B). By 2017, there were no differences in dormant pruning weights across fumigation treatments ( $p=0.233$ ) (Figure 2C). Yield was first collected in 2017, three years postfumigation. There was no effect of fumigation on vine yield (data not shown, $p=0.653$ ). Average yield was $2.72 \mathrm{~kg} / \mathrm{vine}$ and ranged from $2.32 \mathrm{~kg} / \mathrm{vine}$ to $3.14 \mathrm{~kg} / \mathrm{vine}$.

Effect of rootstock on vine vigor. Rootstock had a significant effect on pruning weights in all three years $(2015$, $p=0.004 ; 2016, p<0.0001 ; 2017, p<0.0001)$. Harmony was the most vigorous rootstock in the first three years of dormant pruning regardless of the relative number of M. hapla $\mathrm{J} 2$ in the soil (Figure 3). There were no differences among the self-grafted, own-rooted, 1103 P, 101-14 Mgt, and Teleki 5C in the first dormant pruning in 2015 (Figure 3A), but some separation between dormant pruning weights were beginning to emerge by 2016 (Figure 3B). In the third year, 2017, most of the rootstocks had higher pruning weights than the selfgrafted and own-rooted vines (Figure 3C), though Teleki 5C was not significantly different from the own-rooted control. Rootstock also had a significant effect on vine yield in 2017, the first year that yield data were collected ( $p=0.003$; Figure 4). This was a partial-cropping year, as the 3 -year-old vines were still too young to crop at their full potential. Vines planted on Harmony rootstock had the highest yield in this partial-cropping year at $3.1 \mathrm{~kg} /$ vine and Teleki $5 \mathrm{C}$ and 101-14 Mgt had the lowest, at 2.3 and $2.4 \mathrm{~kg} / \mathrm{vine}$, respectively. 1103 $\mathrm{P}$, self-grafted, and own-rooted vine yields were intermediate (Figure 4). The difference from the lowest to highest average yields across rootstock treatments was $2.25 \mathrm{t} / \mathrm{ha}$ ( 1 ton/acre).

Effect of fumigation and rootstock on $M$. hapla. There were no differences in M. hapla J2 population densities in soil between plots in fall 2014 prior to establishing the experiment, with an average of $69 \mathrm{M}$. hapla J2/250 g soil (Table 1). In spring 2015 (6 months postfumigation/preplant), there was a fumigation effect $(p=0.002)$; fumigated plots had zero $M$. hapla $\mathrm{J} 2$ compared to nonfumigated plots, which had on average $28 \mathrm{M}$. hapla J2/250 g soil. There was no rootstock effect in spring 2015; this was expected as vines were not planted until after the spring 2015 sampling. However, at all time periods after spring 2015, the self-grafted and ownrooted vines had higher $M$. hapla J2 population densities than the rootstocks (Table 1). In fall 2015 (one year postfumigation/six months postplanting), fumigation, rootstock, and fumigation $\times$ rootstock were all significant. Looking more closely at the variables, the differences in M. hapla $\mathrm{J} 2$ population densities within both the susceptible own-rooted and self-grafted Chardonnay treatments were mostly due to 
fumigation, as the differences in initial nematode density as a result of soil fumigation were amplified as the nematode reproduced. Conversely, there was little difference in M. hapla density among F, NF, and NF+ within the rootstock treatments
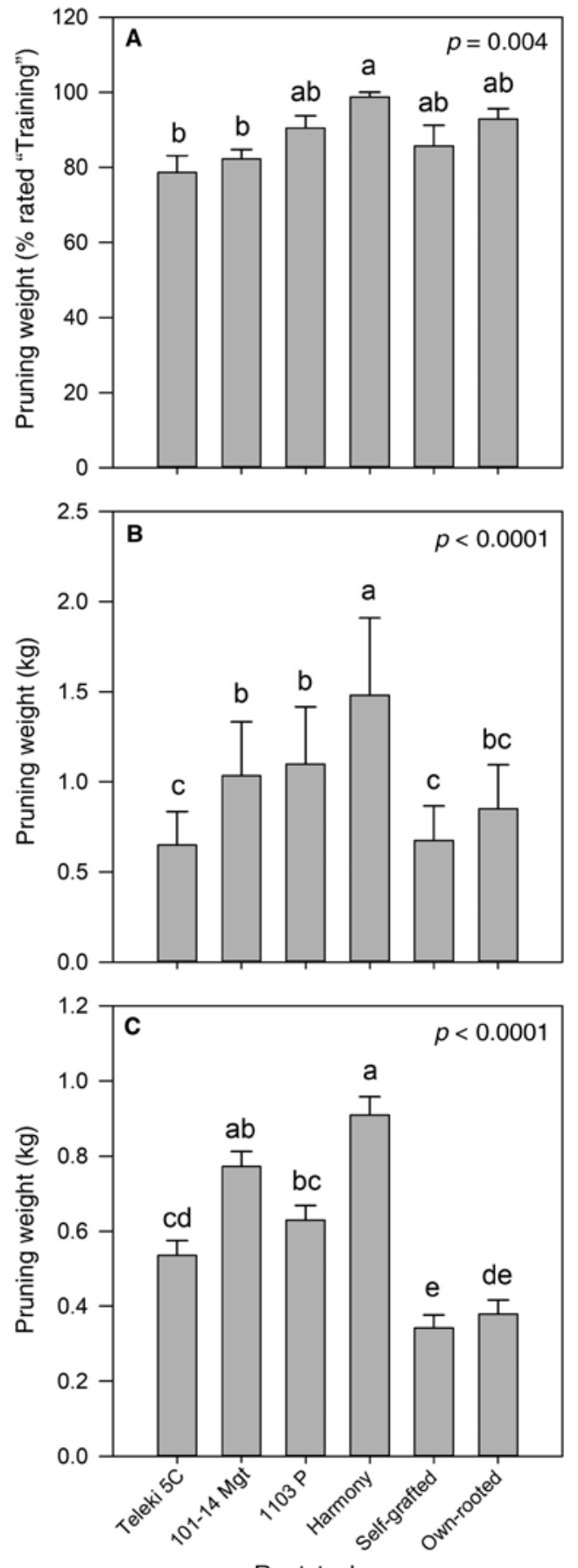

Rootstock

Figure 3 Dormant vine pruning weights by rootstock treatment in (A) 2015, (B) 2016, and (C) 2017. Rootstocks were Teleki 5C, 101-14 Millardet et de Grasset (101-14 Mgt), Paulsen 1103 (1103 P), Harmony, self-grafted Vitis vinifera Chardonnay (Self-grafted), and own-rooted nongrafted Chardonnay (Own-rooted). All rootstocks were grafted with $V$. vinifera Chardonnay Foundation Plant Services selection 15 as the scion. Fumigation treatments were combined within rootstock treatments as there was no rootstock $\times$ fumigation interaction. Within a graph, different letters denote significant differences among treatment means at $\alpha=0.05$ using Tukey's honest significant difference. Error bars are standard error $(n=4)$. Fumigation occurred on 18 Sept 2014 and planting occurred on 12 May 2015.
( $p=0.0001)$, but a large difference between rootstocks and the controls. When all data were considered together, fumigation, rootstock, and fumigation $\times$ rootstock were all significant.

By spring 2016 (1.5 years postfumigation/1 year postplanting), the effect of fumigation on M. hapla J2 population densities was lost $(p=0.072)$, and only rootstock had a significant effect (Table 1). In spring 2016, one year postplanting, Harmony and Teleki $5 \mathrm{C}$ had fewer M. hapla $\mathrm{J} 2$ than the own-rooted control $(p=0.005)$, and the other two rootstocks were no different from either the self-grafted or own-rooted vines. In fall 2016, Harmony, Teleki 5C, 1103 P, and 101-14 Mgt vines all supported fewer M. hapla J2 $(p=0.001)$ and eggs $(p<0.0001)$ (Figure 5A) than the self-grafted and own-rooted controls. Although the effect of fumigation treatment on M. hapla J2 population densities was not significant in fall 2016, it was a factor for M. hapla egg densities per $\mathrm{g} \operatorname{root}(p=0.0001)$, with $\mathrm{NF}+$ having more eggs than NF and $\mathrm{F}$ treatments (Figure 5B).

In spring 2017, Teleki 5C and 1103 P rootstocks supported fewer $M$. hapla $\mathbf{J} 2$ than own-rooted and self-grafted vines $(p<0.0001)$ (Table 1). In fall 2017, Harmony, Teleki 5C, 1103 $\mathrm{P}$, and 101-14 Mgt vines all supported fewer M. hapla J2 than the own-rooted control $(p<0.0001)$, but only Teleki $5 \mathrm{C}$ and Harmony had fewer M. hapla eggs than own-rooted and selfgrafted vines $(p<0.0001)$ (Figure 5C). There was no effect of fumigation on $M$. hapla egg densities, though $\mathrm{NF}+$ had higher densities of $M$. hapla eggs than F or NF treatments ( $p=0.003$ ) (Figure 5D). By spring 2018 (3.5 years postfumigation/3 years postplanting), all plots had measurable population densities of M. hapla $\mathrm{J} 2$, though nematode density differed by rootstock

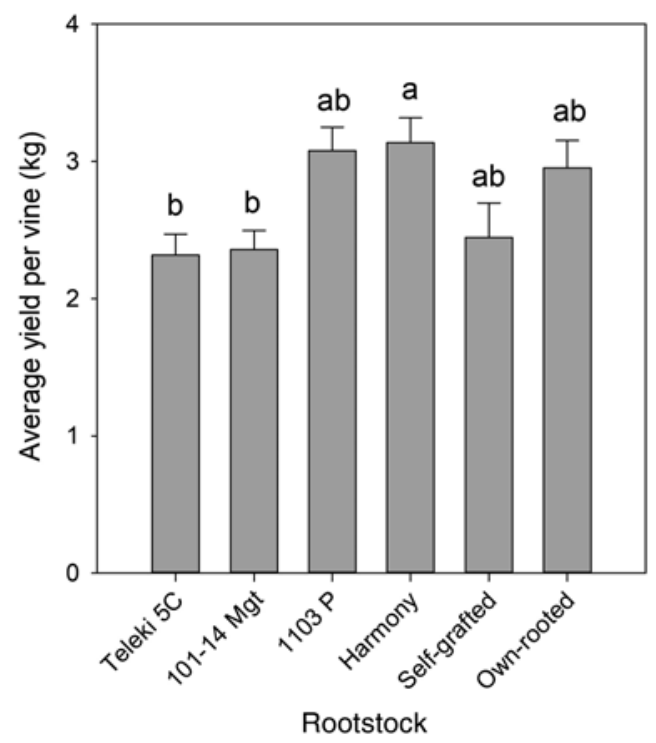

Figure 4 First partial harvest in 2017 with average yield per vine by rootstock treatment $(p=0.003)$. Rootstocks were Teleki $5 \mathrm{C}, 101-14$ Millardet et de Grasset (101-14 Mgt), Paulsen 1103 (1103 P), Harmony, self-grafted Vitis vinifera Chardonnay (Self-grafted), and own-rooted nongrafted Chardonnay (Own-rooted). All rootstocks were grafted with V. vinifera Chardonnay Foundation Plant Services selection 15 as the scion. Fumigation treatments were combined within rootstock treatments as there was no rootstock $\times$ fumigation interaction. Within a graph, different letters denote significant differences among treatment means at $\alpha=$ 0.05 using Tukey's honest significant difference. Error bars are standard error $(n=4)$. Fumigation occurred on 18 Sept 2014 and planting occurred on 12 May 2015. 
alone $(p<0.0001)$ (Table 1$)$. Teleki $5 \mathrm{C}$ had the fewest $M$. hapla $\mathrm{J} 2$ and the own-rooted control the most, with little difference among the other treatments $(p<0.0001)$ (Table 1). In general, $M$. hapla population densities slowly increased over time in all treatments.

Nematode density category. The trends described above and in Table 1 for M. hapla J2 population dynamics over time were better visualized by categorically describing $M$. hapla $\mathrm{J} 2$ (Figure 6). In own-rooted and self-grafted Chardonnay vines, only the F treatment in the first year (fall 2015) resulted in the lowest density of M. hapla (Category 1, $<50$ M. hapla J2/250 $\mathrm{g}$ soil); $\mathrm{NF}$ and $\mathrm{NF}+$ treatments had moderate to high population densities (Category 2, 50 to 150 M. hapla J2/250 g soil; Category 3, >150 M. hapla J2/250 g soil). By fall of 2016 and 2017, M. hapla population densities on both treatments with $V$. vinifera roots were in Category 3, regardless of fumigation treatment. Conversely, M. hapla population densities never exceeded Category 1 in Teleki 5C vines. For 101-14 Mgt and 1103 P rootstocks, $M$. hapla population densities eventually reached Categories 2 and 3 but not until fall 2017. Harmony rootstock in fumigated plots had $M$. hapla densities in Category 1 through fall 2017. In NF+ plots, Harmony did support higher M. hapla population densities, with densities increasing from Category 2 in fall of 2015 to Category 3 in fall of 2017. Overall, M. hapla population densities increased in all the rootstock treatments over the first three years of establishment, just at a slower rate than in the own-rooted and self-grafted controls.

Ranked pruning weights in own-rooted vines relative to M. hapla population densities. Given that the more common practice in Washington State is to grow $V$. vinifera on its own roots, we evaluated whether there was a relationship between M. hapla $\mathrm{J} 2$ population density and vine vigor (measured as pruning weight) using linear regression and ranked variables. There was a similar negative trend between ranked M. hapla population densities and ranked pruning weights in both of the full vegetative growth cycles considered (cycle 1: influence of M. hapla $\mathrm{J} 2$ population densities in fall 2015 on subsequent vine growth in 2016, recorded as fall 2016 pruning weights; and cycle 2: influence of $M$. hapla $\mathrm{J} 2$ population densities in fall 2016 on subsequent vine growth, recorded as fall 2017 pruning weights). Pruning weights of own-rooted vines decreased as M. hapla J2 population densities increased (cycle 1: $\mathrm{R}^{2}=0.34, p=0.003$; cycle $2: \mathrm{R}^{2}=0.30, p=0.006$ ) (Figure 7). The regression lines for both sets of years are nearly parallel, with very similar trends. This is evidence for there being a negative relationship between $M$. hapla population density and vine growth in the subsequent year.

Nematode dosage. Nematode dosage quantifies the accumulated impact of nematode pressure over time (Noling

Table 1 Average Meloidogyne hapla second-stage juvenile (J2) soil densities (per $250 \mathrm{~g}$ soil) at fall and spring sampling dates from fall 2014 to spring 2018. Fumigation occurred on 18 Sept 2014 and planting on 12 May 2015. Fumigation treatments were fumigated (F), nonfumigated (NF), and nonfumigated inoculated (NF+) with $\sim 20,000$ additional $M$. hapla eggs applied at planting.

Rootstocks were Teleki 5C (5C), 101-14 Millardet et de Grasset (101-14 Mgt), Paulsen 1103 (1103 P), Harmony, self-grafted Vitis vinifera Chardonnay (self-grafted), and own-rooted nongrafted Chardonnay (own-rooted). All rootstocks were grafted with $V$. vinifera Chardonnay Foundation Plant Services selection 15 as the scion.

\begin{tabular}{|c|c|c|c|c|c|c|c|c|c|c|c|c|c|c|c|c|}
\hline \multirow{3}{*}{$\begin{array}{l}\text { Rootstock/ } \\
\text { fumigation }\end{array}$} & \multicolumn{16}{|c|}{ Timing of sampling } \\
\hline & \multicolumn{2}{|c|}{ Fall 2014} & \multicolumn{2}{|c|}{ Spring 2015} & \multicolumn{2}{|c|}{ Fall 2015} & \multicolumn{2}{|c|}{ Spring 2016} & \multicolumn{2}{|c|}{ Fall 2016} & \multicolumn{2}{|c|}{ Spring 2017} & \multicolumn{2}{|c|}{ Fall 2017} & \multicolumn{2}{|c|}{ Spring 2018} \\
\hline & Mean & SE & Mean & SE & Mean & SE & Mean & $\overline{\text { SE }}$ & Mean & SE & Mean & $\overline{\text { SE }}$ & Mean & SE & Mean & SE \\
\hline \multicolumn{17}{|l|}{ Own-rooted } \\
\hline $\mathrm{F}$ & 41 & 25 & 0 & 0 & 25 & 23 & 45 & 33 & 225 & 154 & 248 & 165 & 240 & 49 & 413 & 218 \\
\hline NF & 83 & 33 & 48 & 35 & 169 & 39 & 180 & 162 & 199 & 93 & 375 & 249 & 210 & 128 & 180 & 107 \\
\hline $\mathrm{NF}+$ & 83 & 33 & 48 & 35 & 338 & 89 & 210 & 82 & 844 & 667 & 143 & 52 & 220 & 106 & 218 & 149 \\
\hline \multicolumn{17}{|l|}{ Self-grafted } \\
\hline $\mathrm{F}$ & 15 & 7 & 0 & 0 & 17 & 8 & 15 & 17 & 281 & 167 & 98 & 57 & 675 & 246 & 713 & 284 \\
\hline NF & 85 & 68 & 45 & 29 & 136 & 85 & 135 & 114 & 461 & 258 & 570 & 338 & 555 & 222 & 780 & 406 \\
\hline $\mathrm{NF}+$ & 85 & 68 & 45 & 29 & 281 & 51 & 165 & 107 & 1058 & 431 & 405 & 210 & 600 & 147 & 514 & 240 \\
\hline \multicolumn{17}{|l|}{ Harmony } \\
\hline $\mathrm{F}$ & 146 & 29 & 0 & 0 & 0 & 0 & 0 & 0 & 0 & 0 & 0 & 0 & 0 & 0 & 30 & 24 \\
\hline NF & 123 & 111 & 28 & 27 & 8 & 10 & 0 & 0 & 150 & 173 & 8 & 9 & 15 & 17 & 98 & 91 \\
\hline $\mathrm{NF}+$ & 123 & 111 & 28 & 27 & 58 & 23 & 0 & 0 & 56 & 65 & 90 & 14 & 320 & 214 & 225 & 88 \\
\hline \multicolumn{17}{|l|}{ 101-14 Mgt } \\
\hline $\mathrm{F}$ & 124 & 45 & 0 & 0 & 41 & 30 & 15 & 17 & 0 & 0 & 60 & 69 & 68 & 30 & 218 & 152 \\
\hline NF & 60 & 35 & 17 & 10 & 4 & 5 & 75 & 66 & 0 & 0 & 98 & 82 & 330 & 236 & 75 & 46 \\
\hline $\mathrm{NF}+$ & 60 & 35 & 17 & 10 & 25 & 12 & 45 & 17 & 11 & 13 & 30 & 35 & 195 & 71 & 195 & 54 \\
\hline \multicolumn{17}{|l|}{$5 C$} \\
\hline F & 25 & 10 & 0 & 0 & 4 & 5 & 45 & 52 & 0 & 0 & 0 & 0 & 20 & 20 & 8 & 9 \\
\hline NF & 18 & 20 & 21 & 5 & 12 & 9 & 0 & 0 & 15 & 17 & 0 & 0 & 0 & 0 & 30 & 35 \\
\hline $\mathrm{NF}+$ & 18 & 20 & 21 & 5 & 21 & 12 & 0 & 0 & 34 & 39 & 30 & 35 & 30 & 20 & 68 & 9 \\
\hline \multicolumn{17}{|l|}{1103 P } \\
\hline $\mathrm{F}$ & 121 & 103 & 0 & 0 & 0 & 0 & 30 & 35 & 0 & 0 & 0 & 0 & 60 & 69 & 53 & 50 \\
\hline NF & 43 & 38 & 7 & 5 & 8 & 6 & 0 & 0 & 0 & 0 & 0 & 0 & 0 & 0 & 53 & 30 \\
\hline $\mathrm{NF}+$ & 43 & 38 & 7 & 5 & 41 & 29 & 75 & 44 & 11 & 13 & 30 & 35 & 180 & 185 & 128 & 59 \\
\hline
\end{tabular}


and Ferris 1987). In the statistical model, both fumigation and rootstock were significant at all time points postplanting $(p \leq 0.0001)$, and there was no significance to the interaction between the two $(p>0.5)$. By fall 2016, after two growing seasons, both the self-grafted and own-rooted vines had a higher cumulative nematode dosage than the vines on rootstocks (Figure 8A), and the gap between the self-grafted and own-rooted vines and rootstocks increased through spring 2018 . The initial nematode density variation caused by fumigation treatments persisted through spring $2018(p<0.0001)$ (Figure 8B).

Effect of fumigation and rootstock on $X$. americanum population densities. There was no difference in $X$. americanum population densities between plots prior to establishing the experiment ( $p=0.53$ ) (Table 2), and the average initial density of $X$. americanum was 214 individuals $/ 250 \mathrm{~g}$ soil. Fumigation was the only significant parameter in the model; there was no difference in $X$. americanum population densities between the rootstocks and own-rooted and self-grafted vines. All rootstocks, regardless of parentage, were able to support the development of the $X$. americanum at this site. Population densities of $X$. americanum varied from season to season in the nonfumigated plots, but these were not related to the rootstock genotype $(p>0.21)$. Fumigation significantly reduced $X$. americanum population densities to near zero from spring 2015 through spring 2018. In fumigated plots, $X$. americanum still had not reached prefumigation densities by spring 2018 .
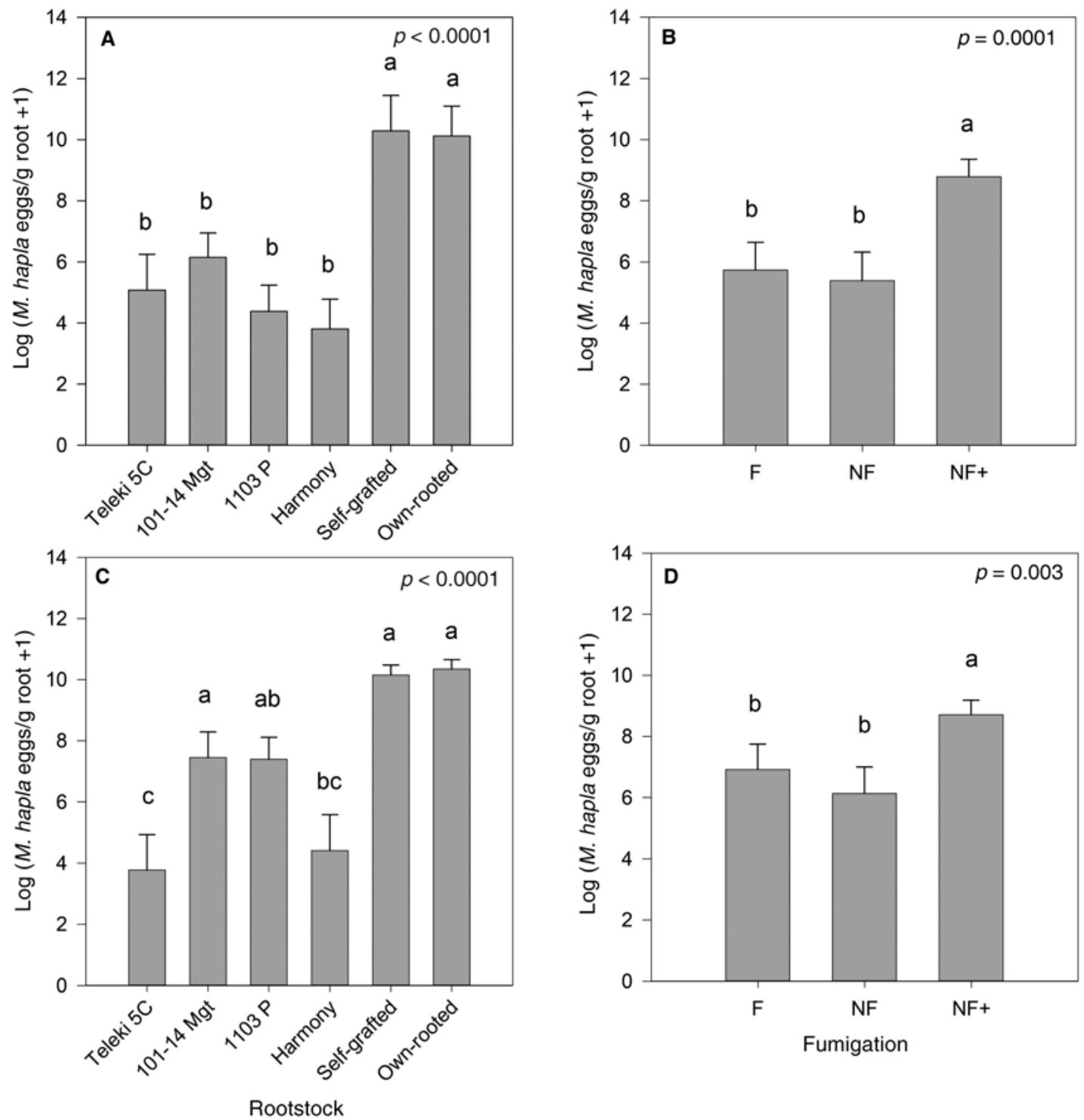

Figure 5 Meloidogyne hapla egg population densities (per g root) in (A) fall 2016 by rootstock, (B) fall 2016 by fumigation, (C) fall 2017 by rootstock, and (D) fall 2017 by fumigation. There was no interaction between rootstocks and fumigation, so they are presented separately. Rootstocks were Teleki 5C, 101-14 Millardet et de Grasset (101-14 Mgt), Paulsen 1103 (1103 P), Harmony, self-grafted Vitis vinifera Chardonnay (Self-grafted), and ownrooted nongrafted Chardonnay (Own-rooted). All rootstocks were grafted with $V$. vinifera Chardonnay Foundation Plant Services selection 15 as the scion. Fumigation treatments were fumigated with metam sodium (F), nonfumigated (NF), and nonfumigated inoculated (NF+) with $\sim 20,000$ additional M. hapla eggs applied at planting. Fumigation occurred on 18 Sept 2014 and planting and additional nematode inoculation (NF+) occurred on 12 May 2015. Error bars are standard error $(n=4)$. Different letters denote significant differences among treatment means at $\alpha=0.05$ using Tukey's honest significant difference. 


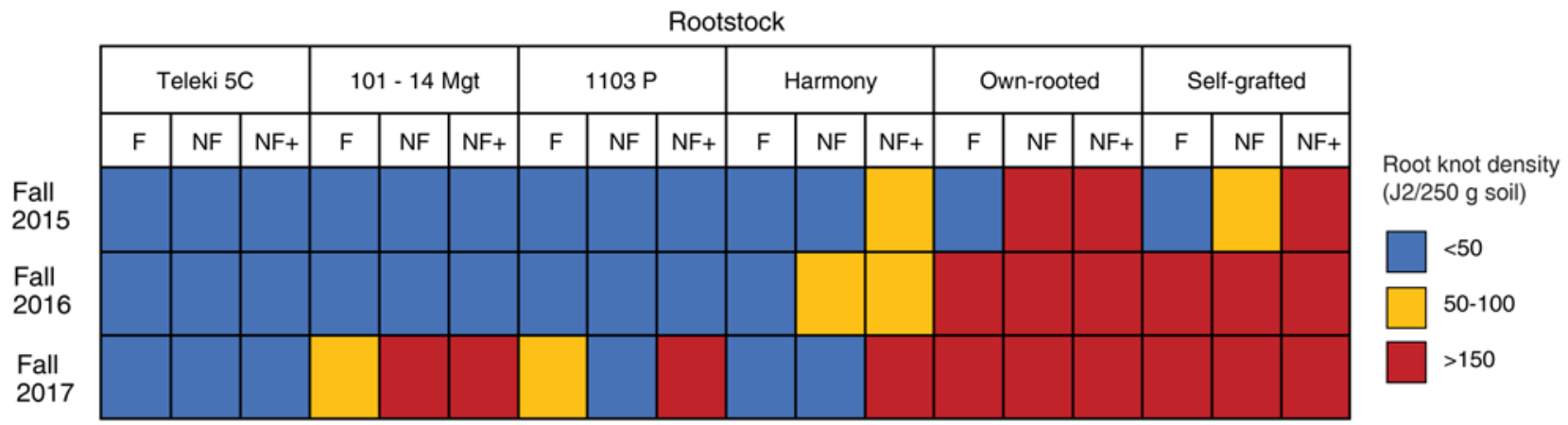

Figure 6 Categorical diagram of Meloidogyne hapla (root-knot nematode) management risk over time. Rootstock and fumigation combination M. hapla second-stage juveniles (J2) density categories: 1) less than $50 \mathrm{M}$. hapla J2/250 g soil (blue; below management threshold), 2) 50 to $150 \mathrm{M}$. hapla J2/250 $\mathrm{g}$ soil (yellow; around proposed management threshold), and 3) more than $150 \mathrm{M}$. hapla J2/250 g soil (red; above management threshold)) in fall of 2015, 2016, and 2017. Fumigation treatments were fumigated (F), nonfumigated (NF), and nonfumigated inoculated (NF+) with 20,000 additional M. hapla eggs at planting. Rootstocks were Teleki 5C, 101-14 Millardet et de Grasset (101-14 Mgt), Paulsen 1103 (1103 P), Harmony, own-rooted nongrafted Vitis vinifera Chardonnay (Own-rooted), and self-grafted $V$. vinifera Chardonnay (Self-grafted). Each data point within a treatment combination is the average of $n=4$. Fumigation occurred on 18 Sept 2014 and planting and additional nematode inoculation occurred on 12 May 2015.

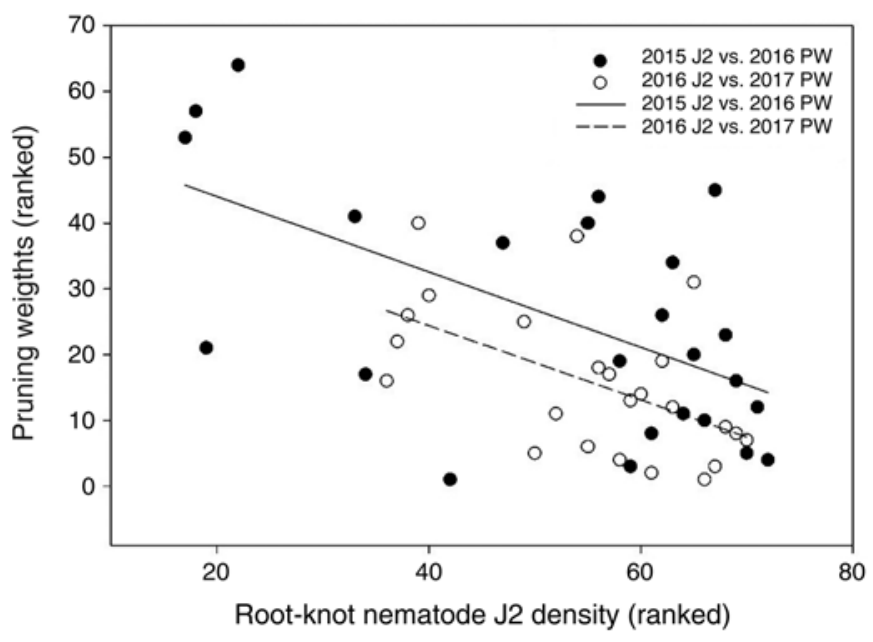

Figure 7 Higher nematode population densities in the previous growing season negatively impact vine vigor (pruning weights; PW) the following season. Linear regression comparison between ranked PW of vines on susceptible Vitis vinifera Chardonnay roots and the previous year's ranked Meloidogyne hapla second-stage juveniles (J2) population densities for two growing season cycles: fall $2015 \mathrm{M}$. hapla J2 densities compared to fall 2016 PW (black circles), and fall 2016 M. hapla J2 densities compared to fall 2017 PW (white circles). 2015 to 2016 regression (solid line): $\mathrm{y}=$ $67.3-0.5846 \mathrm{x}, \mathrm{R}^{2}=0.34, p=0.003 .2016$ to 2017 regression (dashed line): $\mathrm{y}=63.7-0.5259 \mathrm{x}, \mathrm{R}^{2}=0.29, p=0.006$.

\section{Discussion}

Over the lifetime of a vineyard, a slow decline in individual vines and vineyard productivity as a result of nematode plant parasitism can significantly reduce farm profits (Raski 1986). In most cases, the bulk of nematode management is focused on preplant soil fumigation. This is the case in Washington vineyards where highly susceptible own-rooted $V$. vinifera is almost exclusively planted (Howland et al. 2015). Our experiment is one of the first investigations (Moyer et al. 2017) on the long-term effectiveness of preplant nematode management tools such as fumigation and rootstocks in Washington vineyards. The winegrape industry in the state is relatively young, and to this point, has been able to plant new vineyards on nonvineyard soils; however, a period of vineyard replanting is approaching and with it, the need for information on managing a potential replant disorder due to plant-parasitic nematodes.

The effects of fumigation and rootstock were complicated with respect to $M$. hapla management. Fumigation reduced $M$. hapla compared to the nonfumigated treatment for the first six months postfumigation. However, by one year postfumigation, there was no difference in $M$. hapla J2 population densities between fumigated and nonfumigated plots, and only the plots inoculated with extra $M$. hapla eggs (NF+) had higher population densities. Even with the added M. hapla, there was no difference among any treatments in $M$. hapla $\mathrm{J} 2$ population densities by 1.5 years postfumigation. These results indicate that preplant soil fumigation is likely not a long-term solution for the management of M. hapla in scenarios where the trellis infrastructure is retained and the fumigant is applied through the existing driplines. The juvenile and adult female life stages of this sedentary endoparasitic nematode's life cycle are embedded within vine roots that are relatively impervious to fumigants at commercial application rates (McKenry et al. 1977), thus preventing their ability to provide complete control of Meloidogyne spp. In addition, the effectiveness of fumigation relies on product distribution throughout the soil profile. This distribution is affected by soil type, temperature, product solubility, and application rate (Ajwa and Trout 2004). M. hapla is generally concentrated under drip emitters in Washington vineyards, where most fine roots are located (Howland et al. 2014), and therefore would likely be concentrated in this area if vines are replanted into the same spot. In addition, the retained trellis system may have a "shadowing" effect, where pockets of soil around the in-ground posts may remain untreated. Altogether, in a situation where the existing vineyard infrastructure is maintained in a replant scenario, any $M$. hapla that are missed by fumigation will likely be in the vicinity of any newly planted vines, increasing the likelihood of reinfestation. Even though fumigation was not effective long-term against $M$. hapla, we cannot discount the 
short-term benefits that this practice might provide; however, these benefits are difficult to measure.

One year postfumigation (six months postplanting), the differences in $M$. hapla densities transitioned from being a result of fumigation to a result of rootstock choice. All the non-vinifera rootstocks evaluated here eventually supported measurable densities of $M$. hapla by the end of the establishment period. This demonstrates that while these rootstocks
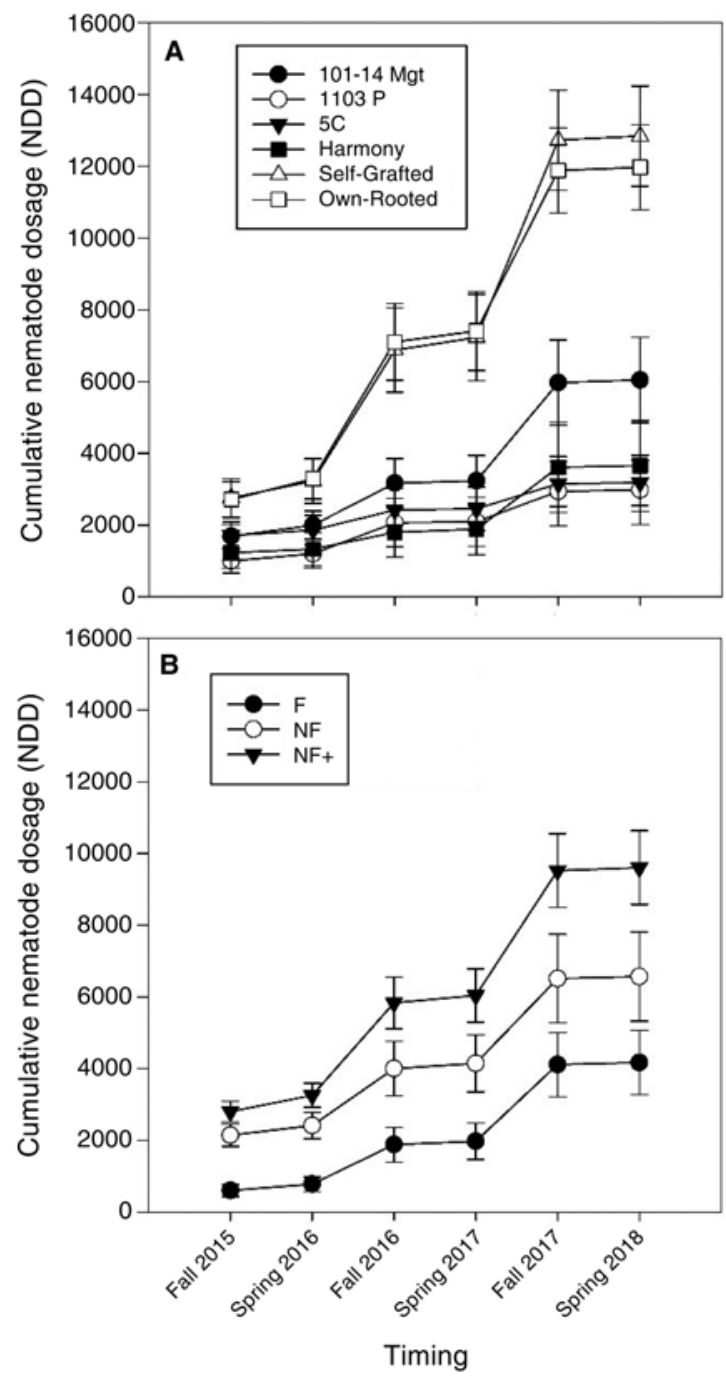

Figure 8 Cumulative Meloidogyne hapla second-stage juvenile (J2) dosage (in nematode degree days; Noling and Ferris 1987) at sampling time points postplanting (spring 2015). Nematode dosage due to rootstock is far lower on rootstocks than the susceptible Vitis vinifera controls $(\mathbf{A})$, and dosage due to fumigation persists across all three years (B). Each error bar is constructed using 1 standard error from the mean. Fumigation occurred on 18 Sept 2014 and planting and additional nematode inoculation occurred on 12 May 2015. Error bars are standard error $(n=4)$. Nematode dosage is a measure of cumulative nematode pressure experienced over time, which is especially important in a perennial system. Rootstocks were Teleki 5C (5C), 101-14 Millardet et de Grasset (101-14 Mgt), Paulsen 1103 (1103 P), Harmony, self-grafted $V$. vinifera Chardonnay (Self-grafted), and own-rooted nongrafted Chardonnay (Own-rooted). All rootstocks were grafted with $V$. vinifera Chardonnay Foundation Plant Services selection 15 as the scion. Fumigation treatments were fumigated with metam sodium (F), nonfumigated (NF), and nonfumigated inoculated (NF+) with 20,000 additional M. hapla eggs applied at planting. Fumigation occurred on 18 Sept 2014 and planting occurred on 12 May 2015. are not resistant to $M$. hapla, they are likely less-than-optimal hosts and may support a smaller density of this nematode while not displaying a typical decline in vigor or yield. These results are similar to what was found by Zasada et al. (2019) in a glasshouse study using an M. hapla population from eastern Washington. In that experiment, M. hapla had a reproduction factor of less than 1 on Harmony and 101-14, indicating that these are poor hosts for $M$. hapla. The own-rooted $V$. vinifera Riesling control, on the other hand, had a reproductive factor of 20.7, indicating that it was an excellent host for $M$. hapla. Though these rootstocks were poor hosts for $M$. hapla in the short-term glasshouse experiment, in the field they were less-than-optimal hosts, as $M$. hapla reproduced on these rootstocks, though far less than on vinifera roots. Of the evaluated rootstocks, Teleki $5 \mathrm{C}$ may be the poorest host for $M$. hapla, as this rootstock was associated with reduced eggs per gram root and, after three years of the experiment, did not support a nematode density that would trigger a management action (Figure 6). Reductions in Meloidogyne arenaria egg densities have been seen previously in Teleki 5C and were associated with poor development of giant cells in vine roots (Anwar and McKenry 2002). We chose the other rootstocks in this study-1103 P, 5C, 101-14 Mgt, and Harmony-because some have demonstrated resistance to at least one Meloidogyne species, including $M$. arenaria, $M$. javanica, and $M$. incognita (Sauer 1967, Chitambar and Raski 1984, Nicol et al. 1999, McKenry et al. 2001, 2006, Ferris et al. 2012). Some resistance-breaking pathotypes of $M$. incognita and $M$. arenaria can reproduce on Harmony but not on other rootstocks, including 101-14 Mgt (Ferris et al. 2012). Resistance to one species of nematode within a genus does not always extrapolate to other species within that genus or even populations within a species (Cain et al. 1984), and few grape rootstocks have been specifically tested against M. hapla.

We used three different approaches to data analysis to further explore the effect of M. hapla on vine productivity and ability to colonize newly planted vines. It is difficult in perennial systems to ascribe the specific impacts of nematode parasitism on overall vine vigor. Unlike in annual systems, there is the compounding of plant age, nematode population changes, and other stresses over the year that make it difficult to measure. One way of accounting for multiple years of nematode pressure may be to sum the cumulative nematode dosage experienced by the vine over time. Plant diseases are often quantified as the disease intensity over time by calculating the AUDPC. Similarly, Noling and Ferris (1987) used female Meloidogyne population densities as a measure of nematode dosage and growing degree days as thermal time. We have used the same premise, with M. hapla J2 population densities as the nematode intensity at each sampling time point (Figure 8). The difference in cumulative nematode dosage from the initial fumigation treatments persisted through spring 2018, which contrasts with the results from the discrete analysis of population densities at each time point (Table 1), where fumigation no longer influenced $M$. hapla densities starting in spring 2016. Though there was no difference between $M$. hapla population densities due to 
fumigation within the sampling points after spring 2016, the lasting effects from there having been a difference in the first year (2015) are better captured using the cumulative nematode dosage and might be more appropriate for a perennial system. Looking at just rootstock (Figure 8A), all rootstocks experienced far lower nematode dosage starting fall 2016, more consistently than the individual sampling date analysis, and the difference in dosage increased over time. It is also interesting to note that the nematode dosage increased mostly from spring to fall and very little in the off-season, from fall to spring.

Secondly, while looking at absolute nematode numbers is useful for statistical analysis, it can be difficult for growers and other end-users to visualize and interpret. A more realistic way of looking at the data is to assign ranges of nematode densities into categories. We chose to use categories around the presumed action threshold of $100 \mathrm{M}$. hapla $\mathrm{J} 2 / 250 \mathrm{~g}$ soil (Santo, unpublished data) (Figure 5). The graphic easily displays both the changes in nematode density due to fumigation and rootstock and shows the changes over time. We used only fall data because that was the most consistent in terms of timing and when growers most commonly sample for nematodes. Interpretations are the same as above, where own-rooted and self-grafted vines were above the threshold in the highest category immediately if not fumigated, unlike most rootstocks evaluated. When fumigated, the effects lasted only through fall 2015 in the own-rooted and self-grafted vines, then the M. hapla population densities were above the threshold by the next year. Teleki 5C, which was the least-suitable host for M. hapla, remained in the lowest population density category through the entire establishment period, below the management threshold.

The third approach was to examine the influence of $M$. hapla on the susceptible own-rooted Chardonnay vines. We altered the initial M. hapla nematode population densities using soil fumigation or through the addition of $M$. hapla eggs at planting. This was intended to measure both the impact of fumigation on $M$. hapla population dynamics and on vine vigor over time. However, in this process, we obtained a wide range of $M$. hapla population densities in the Chardonnay vines grown on their own roots. This gave us an opportunity to see whether there was a relationship between M. hapla population densities and vine vigor. We did find an overall decrease in pruning weights when vines had higher ranked densities of M. hapla in the fall of the previous year. This was consistent over two cycles of fall M. hapla J2 densities and the next year's pruning weight, demonstrating reduced vine vigor with increased $M$. hapla J2 population densities in soil. Since these values are ranked, it is difficult to define a specific nematode density that results in a reduction in pruning weights; this examination was mainly to identify whether there was a pattern of vigor reduction. The $\mathrm{R}^{2}$ values for these regressions were not particularly high $(0.34,0.29)$, but high variability in vine vigor is often common in the early stages of vineyard establishment, regardless of vine health status.

The other plant-parasitic nematode widely distributed in this vineyard, $X$. americanum, was controlled by fumigation with metam sodium; fumigated plots had almost zero $X$. americanum through spring of $2018,3.5$ years postfumigation. $X$. americanum is often found deep in the soil in

Table 2 Average Xiphinema americanum population densities (per $250 \mathrm{~g}$ soil) at fall and spring sampling dates from fall 2014 to spring 2018. Fumigation occurred on 18 Sept 2014, and planting on 12 May 2015. Fumigation treatments were fumigated (F) and nonfumigated (NF). Rootstocks were Teleki 5C (5C), 101-14 Millardet et de Grasset (101-14 Mgt), Paulsen 1103 (1103 P), Harmony, self-grafted Vitis vinifera Chardonnay (self-grafted), and own-rooted nongrafted Chardonnay (own-rooted). All rootstocks were grafted with $V$. vinifera Chardonnay Foundation Plant Services selection 15 as the scion.

\begin{tabular}{|c|c|c|c|c|c|c|c|c|c|c|c|c|c|c|c|c|}
\hline \multirow{3}{*}{$\begin{array}{l}\text { Rootstock/ } \\
\text { fumigation }\end{array}$} & \multicolumn{16}{|c|}{ Timing of sampling } \\
\hline & \multicolumn{2}{|c|}{ Fall 2014} & \multicolumn{2}{|c|}{ Spring 2015} & \multicolumn{2}{|c|}{ Fall 2015} & \multicolumn{2}{|c|}{ Spring 2016} & \multicolumn{2}{|c|}{ Fall 2016} & \multicolumn{2}{|c|}{ Spring 2017} & \multicolumn{2}{|c|}{ Fall 2017} & \multicolumn{2}{|c|}{ Spring 2018} \\
\hline & Mean & SE & Mean & SE & Mean & SE & Mean & SE & Mean & SE & Mean & SE & Mean & SE & Mean & SE \\
\hline \multicolumn{17}{|l|}{ Own-rooted } \\
\hline $\mathrm{F}$ & 253 & 69 & 0 & 0 & 0 & 0 & 0 & 0 & 0 & 0 & 0 & 0 & 0 & 0 & 0 & 0 \\
\hline NF & 149 & 7 & 62 & 26 & 37 & 9 & 0 & 0 & 240 & 123 & 23 & 17 & 75 & 66 & 90 & 49 \\
\hline \multicolumn{17}{|l|}{ Self-grafted } \\
\hline $\mathrm{F}$ & 145 & 58 & 0 & 0 & 0 & 0 & 0 & 0 & 0 & 0 & 0 & 0 & 0 & 0 & 8 & 9 \\
\hline NF & 155 & 79 & 86 & 67 & 37 & 16 & 0 & 0 & 41 & 28 & 68 & 55 & 90 & 45 & 98 & 62 \\
\hline \multicolumn{17}{|l|}{ Harmony } \\
\hline $\mathrm{F}$ & 303 & 125 & 0 & 0 & 0 & 0 & 0 & 0 & 0 & 0 & 0 & 0 & 0 & 0 & 0 & 0 \\
\hline NF & 204 & 78 & 69 & 43 & 25 & 17 & 0 & 0 & 131 & 79 & 83 & 22 & 90 & 66 & 203 & 157 \\
\hline \multicolumn{17}{|l|}{ 101-14 Mgt } \\
\hline $\mathrm{F}$ & 138 & 76 & 0 & 0 & 0 & 0 & 0 & 0 & 0 & 0 & 0 & 0 & 0 & 0 & 8 & 9 \\
\hline $\mathrm{NF}$ & 239 & 57 & 24 & 8 & 33 & 26 & 15 & 17 & 50 & 5 & 90 & 49 & 30 & 35 & 113 & 54 \\
\hline \multicolumn{17}{|l|}{$5 \mathrm{C}$} \\
\hline $\mathrm{F}$ & 203 & 122 & 0 & 0 & 0 & 0 & 0 & 0 & 2 & 2 & 8 & 9 & 0 & 0 & 0 & 0 \\
\hline NF & 299 & 38 & 21 & 14 & 12 & 5 & 30 & 20 & 45 & 52 & 83 & 38 & 240 & 102 & 225 & 67 \\
\hline \multicolumn{17}{|l|}{$1103 \mathrm{P}$} \\
\hline $\mathrm{F}$ & 331 & 245 & 0 & 0 & 0 & 0 & 0 & 0 & 0 & 0 & 0 & 0 & 0 & 0 & 15 & 17 \\
\hline NF & 144 & 42 & 52 & 34 & 37 & 16 & 60 & 40 & 105 & 84 & 83 & 54 & 20 & 20 & 68 & 26 \\
\hline
\end{tabular}


Washington (Howland et al. 2014, East et al. 2019b), and this nematode has a long life cycle of one year or more (Malek 1969). Based upon its biology, it would likely take multiple years for this nematode to recolonize the upper $45 \mathrm{~cm}$ of soil where sampling occurred. $X$. americanum can feed along roots and are likely able to feed on roots that are deeper in the soil, so they may not have incentive to return to the upper profile (Cohn 1970). From a management perspective, fumigation was effective, but the rootstocks evaluated in this experiment would not be effective against $X$. americanum. All the rootstocks evaluated supported high population densities of the $X$. americanum that are found in Washington State, which is consistent with previous research with $X$. americanum in California (McKenry et al. 2004, Ferris et al. 2012).

From a vine vigor perspective, Teleki $5 \mathrm{C}$ was consistently the least vigorous of the rootstocks and Harmony the most vigorous, as determined by dormant pruning weights and yield from the cropping year. Both $1103 \mathrm{P}$ and 101-14 Mgt had intermediate vigor, which does contrast with previous findings in the region (Keller et al. 2012). This matches the vigor categories from California, where Harmony and 1103 $\mathrm{P}$ are medium-to-high vigor, 101-14 Mgt is medium vigor, and Teleki 5C is low-to-medium vigor (Bettiga 2003), in both reproductive (yield) and vegetative (pruning weight) growth. Some of Harmony's vigor in the first year may be attributed to the fact that it was a green graft that had not gone through a dormancy period, unlike the rest of the grafted rootstocks; however, Harmony is known as a high-vigor rootstock and was consistently the most vigorous rootstock in all years. Even in the presence of $M$. hapla, lower vigor rootstocks, like $1103 \mathrm{P}$ and Teleki 5C, maintained vegetative growth, whereas it appeared that the own-rooted and self-grafted vines could not, exacerbated by the loss of the fumigation effect. This relative vigor decline in the own-rooted and self-grafted vines across all soil treatments, especially in year 3 (2017), is likely attributable to $M$. hapla, as it was present in all plots. The high nematode dosage experienced by own-rooted vines compared to rootstocks (Figure 8), as well as the relationship between higher $M$. hapla population density and lower vine vigor the following year (Figure 7), support this hypothesis.

Growth stimulation can also occur in the presence of nematode feeding (Seinhorst 1965), which may explain why vines grown in nonfumigated plots had greater pruning weights than vines in fumigated plots in 2015. This stimulation occurs during light feeding and is overcome under heavy nematode pressure. This effect was weaker in 2016, and no effect was seen in 2017. It is likely that the vigor response seen by year 3 was mostly a result of nematode feeding, as $M$. hapla population densities in the own-rooted controls were far higher than those in the rootstocks. This could be due to both $X$. americanum and $M$. hapla parasitism. McKenry and Anwar (2006) found that $X$. americanum parasitism stimulated growth in 11 of the 16 rootstock cultivars evaluated, and that parasitism by $M$. javanica stimulated growth of Harmony. Growth stimulation may contribute to the tolerance of some rootstocks to nematode parasitism. An additional potential explanation for reduced pruning weights in fumigated plots as compared to nonfumigated may be that fumigation kills not only nematodes in soil, but mycorrhizal fungi that form symbioses with grapevines. Stunting in some vineyards has been found to be a result of poor colonization with mycorrhizae (Menge et al. 1983), and metam sodium has been found to kill mycorrhizal fungi (Davis et al. 1996). This study underlines the importance of understanding which nematode species are present in a vineyard facing replant, as the efficacy of these management techniques (fumigation and rootstocks) are species dependent.

\section{Conclusion}

Plant-parasitic nematode management is an important part in the process of vineyard replanting. Fumigation was not an effective long-term strategy for suppressing $M$. hapla. Soil population densities of this nematode recovered 1.5 years postfumigation and exceeded prefumigation levels, as well as the proposed threshold for management, by two years postfumigation in plots containing own-rooted and self-grafted vines. Increased $M$. hapla $\mathrm{J} 2$ population densities in the fall of a year were correlated with lower pruning weights in the following year in own-rooted and self-grafted Chardonnay vines. All non-vinifera rootstocks had increased vigor over the own-rooted and self-grafted vines by year 3 of establishment and generally supported lower M. hapla population densities compared to $V$. vinifera vines. However, by year 3, all treatments regardless of rootstock genotype had measurable $M$. hapla population densities. Therefore, it appears that none of these non-vinifera rootstocks is fully resistant to M. hapla, but rather, they are better classified as partially resistant to feeding (i.e., poor hosts). Fumigation was an effective tactic for management of $X$. americanum, which was only detectable in fumigated soil 3.5 years postfumigation. None of the rootstocks evaluated (1103 P, 101-14 Mgt, Teleki 5C, and Harmony) was resistant to $X$. americanum, as expected. As Washington growers move forward into an era of replant, adoption of rootstocks for $M$. hapla management is a potentially viable option, whereas fumigation may provide only short-term protection from $M$. hapla but is a suitable management strategy for the long-term suppression of $X$. americanum.

\section{Literature Cited}

Ajwa HA and Trout T. 2004. Drip application of alternative fumigants to methyl bromide for strawberry production. HortScience 39:1707-1715.

Anwar SA and McKenry MV. 2002. Developmental response of a resistance-breaking population of Meloidogyne arenaria on Vitis spp. J Nematol 34:28-33.

Bettiga LJ. 2003. Wine Grape Varieties in California. Publication 3419. UCANR Publications, Oakland, CA.

Cain DW, McKenry MV and Tarailo RE. 1984. A new pathotype of root-knot nematode on grape rootstocks. J Nematol 16:207-208.

Chitambar JJ and Raski DJ. 1984. Reactions of grape rootstocks to Pratylenchus vulnus and Meloidogyne spp. J Nematol 16:166-170.

Cohn E. 1970. Observations on the feeding and symptomology of $\mathrm{Xi}$ phinema and Longidorus on selected host roots. J Nematol 2:167-173.

Davis R, Nunez J, Vargas R, Weir B, Wright S and Munier D. 1996. Metam-sodium kills beneficial soil fungi as well as cotton pests. Calif Agr 50:42-44. 
East KE, Zasada IA, Schreiner RP and Moyer MM. 2019a. Developmental dynamics of Meloidogyne hapla in Washington wine grapes. Plant Dis 103:966-971.

East KE, Moyer MM, Madden NM and Zasada IA. 2019b. How low can they go? Plant-parasitic nematode distribution in a Washington vineyard. Catalyst: Discovery into Practice 3:31-36.

Ferris H and McKenry MV. 1975. Relationship of grapevine yield and growth to nematode densities. J Nematol 7:295-304.

Ferris H, Zheng L and Walker MA. 2012. Resistance of grape rootstocks to plant-parasitic nematodes. J Nematol 44:377-386.

Harris AR. 1983. Resistance of some Vitis rootstocks to Xiphinema index. J Nematol 15:405-409.

Howland AD, Schreiner RP and Zasada IA. 2014. Spatial distribution of plant-parasitic nematodes in semi-arid Vitis vinifera vineyards in Washington. J Nematol 46:321-330.

Howland AD, Skinkis PA, Wilson JH, Riga E, Pinkerton JN, Schreiner RP and Zasada IA. 2015. Impact of grapevine (Vitis vinifera) varieties on reproduction of the northern root-knot nematode (Meloidogyne hapla). J Nematol 47:141-147.

Hussey RS and Barker KR. 1973. Comparison of methods of collecting inocula of Meloidogyne spp., including a new technique. Plant Dis Rep 57:1025-1028.

Jenkins WR. 1964. A rapid centrifugal-flotation technique for separating nematodes from soil. Plant Dis Rep 48:692.

Keller M, Mills LJ and Harbertson JF. 2012. Rootstock effects on deficit-irrigated winegrapes in a dry climate: Vigor, yield formation, and fruit ripening. Am J Enol Vitic 63:29-39.

Lembright HW. 1990. Soil fumigation: Principles and application technology. J Nematol 22:632-644.

Malek RB. 1969. Population fluctuations and observations of the life cycle of Xiphinema americanum associated with cottonwood (Populus deltoides) in South Dakota. Proc Helminthol Soc Wash 36:270-274.

McKenry MV and Anwar SA. 2006. Nematode and grape rootstock interactions including an improved understanding of tolerance. J Nematol 38:312-318.

McKenry MV, Thomason IJ and Naylor P. 1977. Dosage-response of root-knot nematode-infected grape roots to cis-1,3-dichloropropene. Phytopathology 67:709-711.

McKenry MV, Kretsch JO and Anwar SA. 2001. Interactions of selected Vitis cultivars with endoparasitic nematodes. Am J Enol Vitic 52:310-316.

McKenry MV, Luvisi D, Anwar SA, Schrader P and Kaku S. 2004. Eight-year nematode study from uniformly designed rootstock trials in fifteen table grape vineyards. Am J Enol Vitic 55:218-227.

Menge JA, Raski DJ, Lider LA, Johnson ELV and Jones NO. 1983. Interactions between mycorrhizal fungi, soil fumigation, and growth of grapes in California. Am J Enol Vitic 34:117-121.
Moyer MM, Mills LM, Hoheisel GA and Keller M. 2011. Assessing and Managing Cold Damage in Washington Vineyards. WSU Extension Publication \#EM042e. Washington State University.

Moyer MM, Boren AN and Tarara JM. 2017. Dual fumigant and herbicide use optimizes replanting preparation in a virus- and nematodeaffected vineyard. Catalyst: Discovery into Practice 1:55-61.

Nicol JM, Stirling GR, Rose BJ, May P and Van Hesswijck R. 1999. Impact of nematodes on grapevine growth and productivity: Current knowledge and future directions, with special reference to Australian viticulture. Aust J Grape Wine Res 5:109-127.

Noling JW and Ferris H. 1987. Nematode-degree days, a density-time model for relating epidemiology and crop losses in perennials. $\mathrm{J}$ Nematol 19:108-118.

Prengaman K. 2019. Washington Vineyard Facing Phylloxera. Good Fruit Grower, 21 Nov 2019. https://www.goodfruit.com/washingtonvineyards-facing-phylloxera/.

Raski DJ. 1986. Plant-parasitic nematodes that attack grapes. In Plant-parasitic Nematodes of Banana, Citrus, Coffee, Grape and Tobacco. pp.43-57. Union Carbide Agricultural Products Company, North Carolina.

Robbins RT. 1993. Distribution of Xiphinema americanum and related species in North America. J Nematol 25:344-348.

Sauer MR. 1967. Root knot tolerance in some grape vine rootstocks. Aust J Exp Agric 7:580-583.

Seinhorst JW. 1962. Modifications of the elutriation method for extracting nematodes from soil. Nematologica 8:117-128.

Seinhorst JW. 1965. The relation between nematode density and damage to plants. Nematologica 11:137-154

Soil Survey Staff, Natural Resources Conservation Service, United States Department of Agriculture. Web Soil Survey. https://websoilsurvey.sc.egov.usda.gov/. Accessed 3/4/2019.

Westphal A, Browne GT and Schneider S. 2002. Evidence for biological nature of the grape replant problem in California. Plant Soil 242:197-203.

Zasada IA, Halbrendt JM, Kokalis-Burelle N, LaMondia J, McKenry MV and Noling JW. 2010. Managing nematodes without methyl bromide. Annu Rev Phytopathol 48:311-328.

Zasada IA, Riga E, Pinkerton JN, Wilson JH and Schreiner RP. 2012. Plant-parasitic nematodes associated with grapevines, Vitis vinifera, in Washington and Idaho. Am J Enol Vitic 63:522-528.

Zasada IA, Howland AD, Peetz AB, East K and Moyer M. 2019. Vitis spp. rootstocks are poor hosts for Meloidogyne hapla, a nematode commonly found in Washington winegrape vineyards. Am J Enol Vitic 70:1-8. 